\title{
Curcumin as Potential Adjuvant Therapy to Prevent Recurrence in Pterygium Excision.
}

\author{
Muhammad Abdurrauf ${ }^{\mathrm{a}}$, Ferdian Ramadhan ${ }^{\mathrm{a}}$, Nurwasis ${ }^{\mathrm{b}}$, Ismi Zuhria ${ }^{\mathrm{b}}$, Evelyn Komaratih ${ }^{\mathrm{b}}$ \\ ${ }^{a}$ Medical Doctor, Ophthalmology Department, Faculty of Medicine, Universitas Airlangga, Surabaya, 60132, \\ Indonesia \\ ${ }^{\mathrm{b}}$ Ophthalmologist (Consultant), Ophthalmology Department, Faculty of Medicine, Universitas Airlangga, Surabaya, \\ 60132, Indonesia
}

\begin{abstract}
Pterygium is a wing-shaped growth from the conjunctiva and fibrovascular tissue onto the surface of the cornea. It has a high postoperative recurrence rate (which can be as high as $89 \%$ and its severity may vary according to the approach adopted and the preoperative conditions). To reduce the postoperative recurrence rate, several surgical techniques have been developed. The pathophysiology of pterygium development is diverse, one of the main factors is the proliferative process initiated by the TGF- $\beta$ mediator. One of the therapies developed to prevent pterygium recurrence is by targeting therapy on the proliferative pathway by adding pterygium surgery with adjuvant MMC therapy. MMC plays a role in inhibiting DNA synthesis, thereby reducing TGF- $\beta$ expression and ultimately inhibiting cell proliferation. Although it can reduce the recurrence rate quite well, MMC has numbers of complications. Therefore, an agent is needed to suppress pterygium proliferation which is relatively safer. Curcumin is a compound derived from the tuber of Curcuma longa L. which includes polyphenols. Curcumin modulates proliferation by stimulating the formation of TGIF which is a negative regulator of TGF- $\beta$ so that it can modulate proliferation.
\end{abstract}

Keyword: pterygium, recurrence, proliferation, curcumin, fibroblast

\section{Introduction}

Pterygium is a growth of fibrovascular tissue from conjunctiva that may pass through cornea. It can cause various degree of discomfort from cosmetics to significantly alter visual function. It affects visual function through disruption of visual axis, corneal induced astigmatism, and tear film alteration. Pterygium grows mainly in equator area called the pterygium belt located between $37^{\circ}$ north latitude to $37^{\circ}$ south latitude. (Hasemi et al., 2017; Coroneo et al., 2019).

surgical procedure is mainstay treatment for pterygium. but, this disease has a excessive postoperative recurrence rate (that's up to $89 \%$ ) and its severity might also vary according to the 
technique adopted and the preoperative conditions). To reduce the postoperative recurrence rate, numerous surgical techniques were developed. the beginning of the pterygium surgical operation approach changed into within the 1900s beginning with the bare sclera approach. This technique has the advantage of being easy to do, but however the recurrence rate when using this approach remains considered excessive (24\%-89\%). This technique is still used today, but numerous clinicians have developed different techniques to reduce the postoperative recurrence rate, such as conjunctival autograft (8.3\%-33\%), amniotic membranes $(3.8 \%-40 \%$ recurrence rate), and with antimetabolites. one of the antimetabolites that is often used is mitomycin C. In therapy with mitomycin $\mathrm{C}$ can lessen the recurrence rate of 7\%-9\% however mitomycin $\mathrm{C}$ has quite a lot of complications. complications of mitomycin $\mathrm{C}$ consist of cataract formation, anterior uveitis, scleral plaque and necrosis, corneal edema and ulceration, anterior chamber inflammation, and nonhealing corneal and scleral defects. because of the numerous complications of mitomycin C, several antimetabolites have started to be investigated for surgical adjuvant therapy options. (Hovanesian et al., 2017; Nuzzi \& Tridicco, 2018)

\section{Pathogenesis pterygium}

Etiology of pterygium is multifactorial but mainly it caused by environment. There were change of concept in pathology from degenerative disease to proliferative disease. Prolonged UV exposure is thought to be one of primary cause of pterygium. UV exposure is affecting on p53 gene which responsible for cell death via apoptosis pathway. The affected cell is more tenacious to cell death and continued to proliferate. (Dushku et al., 2001; Kim et al., 2016)

It is characterized by an increase in TGF- $\beta$ and fibroblasts as key factors in the development of pterygium. The presence of activated fibroblasts in pterygia is likely a result of the action of TGF- $\beta$, given that the expression of this growth factor was reported in pterygium tissue relative to normal conjunctiva and in pterygium fibroblast cultures. This is in contrast to transcriptional expression where increased TGF- $\beta$ mRNA was only reported in the pterygium epithelium but not the subepithelial fraction, except in the case of recurrent pterygium. Unfortunately, several other studies did not differentiate between specific TGF- $\beta$ isoforms so it would be difficult to know whether the staining was due to normal corneal expression of TGF- $\beta 2$ or true upregulation. The expression pattern of TGF- $\beta$ receptors in pterygium is unknown. Nonetheless, blockade of the TGF- $\beta$ system has been targeted by several groups as a possible 
therapy for pterygium with amniotic membrane grafts (which regulate TGF- $\beta$ ) showing some efficacy both in vitro in pterygium fibroblast cultures, and also in the clinical setting. TGF- $\beta$ expresses a signaling response through the Smad pathway and the non-Smad pathway. In the Smad pathway, TGF- $\beta$ will bind to TGF- $\beta$ RII which will activate Smad II/II, then Smad II/III will combine with Smad IV to form a Smad complex which will go to the nucleus to stimulate transcriptional regulation that plays a role in fibrosis. (Horbelt et al., 2012; Cardenas-cantu et al, 2016; Kim et al., 2016)

In pterygium recurrence after excision the mechanism is similar to the primary mechanism of pterygium formation. the wound-healing response of the conjunctiva, and of the anterior segment of the eye in general, is actually similar to that of peripheral nervous system tissue with a primary focus of fibrosis. This response is thought to play a key role in the pathophysiology of several eye conditions. Migration of fibroblast cells plays an important role in the wound healing process. Modulation of fibroblast activity in conjunctival wound healing has the potential to improve the outcome of several eye diseases. Among several eye diseases characterized by scar tissue, there are 3 diseases in which the formation of scar tissue plays a major role. These diseases are ocular cicatrical pemphigoid, vernal keratoconjunctivitis and pterygium. Pterygium is multifactorial and it is thought that the major contributor to the development of pterygium is UV light exposure which causes cellular injury and conjunctival tissue fibrosis. It is characterized by in vitro studies of pterygium fibroblasts showing hyperactivity. (Zada et al., 2017; Ellis et al., 2018)

The mechanism of wound healing in the conjunctiva is more or less similar to the reaction for wound healing elsewhere. When there is an injury, a fibrin clot is formed to close the wound. In addition, the fibrin clot will release growth factors and cytokines. One of the roles is Platelet Derivate Growth Factor (PDGF). PDGF itself will stimulate inflammatory cells such as monocytes, neutrophils and other inflammatory cells to secrete pro-inflammatory cytokines, resulting in an inflammatory process and the formation of fibroblasts. In addition, PDGF also stimulates the formation of VEGF for neovascular formation. (Zada et al., 2017)

Then after inflammation occurs, PDGF will stimulate fibroblasts to secrete TGF- $\beta$ and then autocrine will stimulate itself to turn into active myofibroblasts to proliferate. In addition, myofibroblasts also have high contractility, which is characterized by intracellular a-smooth 
muscle actin activity. This activity increases the expression of extracellular matrix (ECM) proteins and facilitates contractures and wound closure. (Zada et al., 2017)

The final remodeling phase involves maturation of the fibrovascular tissue into a mature scar. It is characterized by the activity of matrix metalloproteinases (MMPs) synthesized by fibroblasts, macrophages, and neutrophils. MMPs mediate selective ECM degradation. Type I collagen replaces type III collagen and is crosslinked and dehydrated to lead to the transformation of cellular granulation tissue into dense hypocellular scars. Reduction of myofibroblast numbers, via apoptosis, is critical for this phase of wound healing. The prolongation of the life of these fibrovascular cells is a major factor causing excessive scarring. (Zada et al., 2017)

Increased TGF- $\beta$ in the proliferative phase of wound healing will increase fibrosis which is the main characteristic for the development of pterygium. TGF- $\beta$ through the samd $2 / 3$ pathway and ERK will convert fibroblasts into proliferating active myofibroblasts. This increase in proliferation is not matched by apoptosis of the cell so it will never reach the remodeling phase. This is caused by pterygium cells that change the characteristics of the p53 gene which is responsible for the apoptotic process. This process causes fibroblasts in the pterygium to continue to proliferate. ((Nakerakanti \& Trojanowska, 2012; Cardenas-cantu et al, 2016; Hu et al., 2018)

\section{Therapy}

Current treatment for pterygium focuses on excision and prevention of recurrence, which is achieved through surgical techniques to cover the bare sclera via transplantation of autologous conjunctiva, limbal tissue, or amniotic membranes. Alternatively, adjuvant chemotherapeutic agents, such as mitomycin $\mathrm{C}$ (MMC), can be applied after excision to prevent cell proliferation (Chui et al., 2008).

Problems associated with MMC are the potential for vision-threatening complications, such as scleral necrosis, infectious scleritis, severe secondary glaucoma, iritis, cataracts, corneal edema, corneal perforation, corneal perforation, and endophthalmitis. (Chui et al., 2008). 


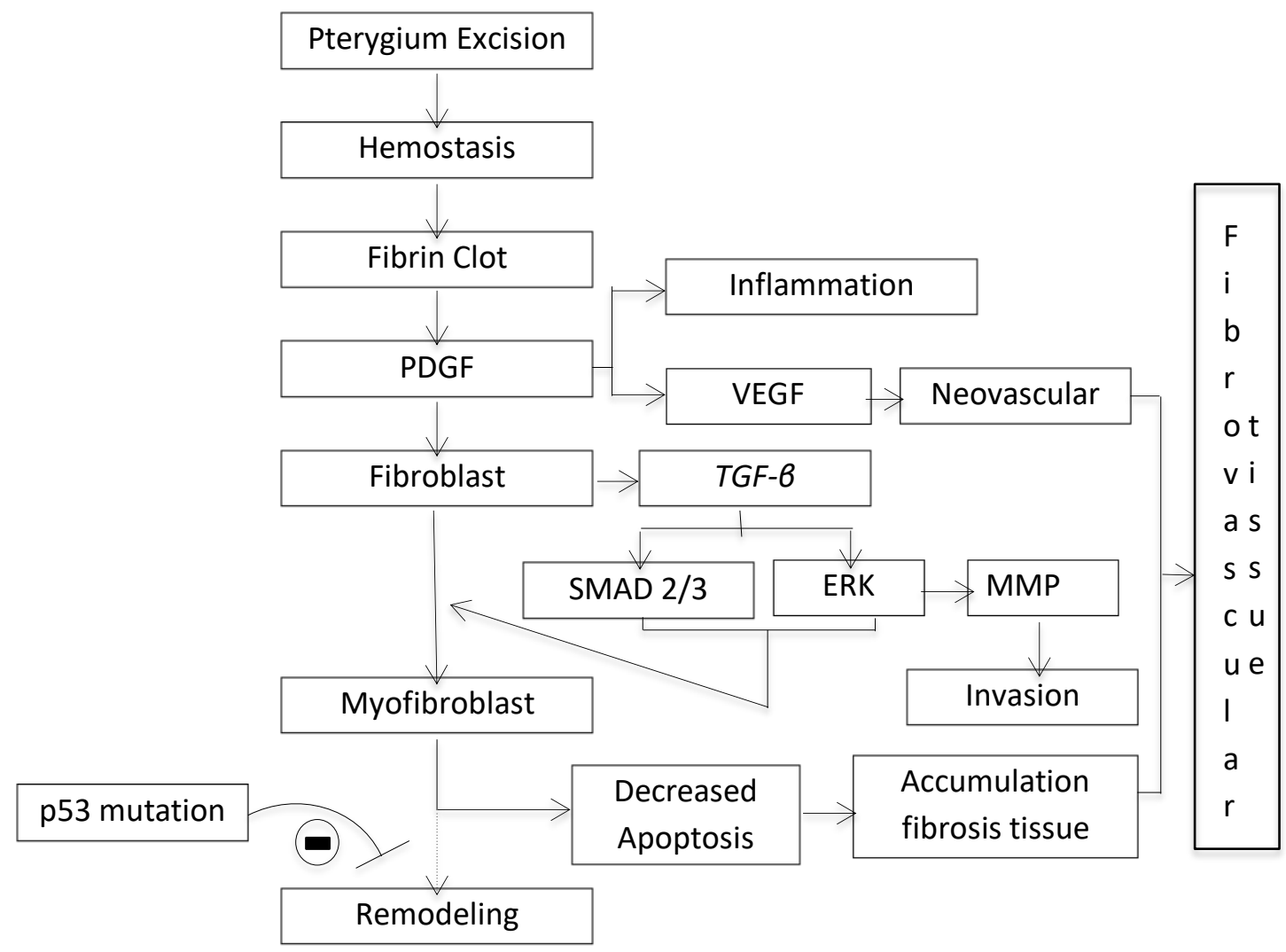

Figure 1. mechanism of pterygium recurrence

Although surgery is the mainstay of treatment for pterygium, an evolving understanding of the pathogenesis of pterygium is leading to the development of future pharmacological interventions. Proliferation of both epithelial and fibrovascular components occurs in pterygium with inflammation being often an exacerbating factor so it makes sense to target the molecules involved in this process as an alternative to the current use of antimetabolites (MMC or 5-FU). (Chui et al., 2008).

\section{Curcumin for potential agent for adjuvant therapy in pterygium}

Curcumin (diferuloylmethane) comes from the tuber Curcuma longa L. which includes polyphenols. Curcumin was isolated more than 140 years ago by Vogel and synthesized in 1913 by Lampe. Curcumin has been used for thousands of years in traditional Chinese medicine and Ayurvedic medicine in Asian countries as an active ingredient in herbal medicines to treat liver disease, rheumatoid disease, diabetes, atherosclerosis, infectious diseases and cancer. Curcumin 
can exert antioxidant, anti-inflammatory, antimutagenic, antimicrobial and anticancer activity effects. Curcumin is very safe and well tolerated substance. Stati et al reported that humans can tolerate up to a dose of $8000 \mathrm{mg}$ per day. (Dorota et al., 2019; Stati et al., 2020)

Curcumin belongs to the group of natural antioxidants. The effect of curcumin on free radicals is carried out by several different mechanisms. These compounds can collect various forms of free radicals, such as ROS and reactive nitrogen species (RNS). Curcumin can also modulate the activity of GSH, catalase, and active SOD enzymes in the neutralization of free radicals. Curcumin can inhibit ROS-producing enzymes, such as lipoxygenase/cyclooxygenase and xanthine hydrogenase/oxidase. Curcumin is a lipophilic compound, which makes it an efficient peroxyl radical scavenger. Like vitamin E, curcumin is also considered a chain-breaking antioxidant. It is proven that curcumin increases GSH levels in both normal and cancer cells. Curcumin suppresses nitric oxide synthase (NOS) activity in murine macrophages as well as NOS synthesis in rat spleen in an in vivo study. It further induces the expression of hemeoxygenase in various cell types. Heme-oxygenase is an enzyme that catalyzes the degradation of heme to biliverdin, iron, and carbon monoxide. NOS also plays an important role in the cellular response to oxidative stress and in angiogenesis. The antioxidant properties of curcumin lead to the inhibition of oxidative stress. This allows people to understand the effectiveness of treatment in various lifestyle-related diseases. (Dorota et al., 2019).

It was reported that curcumin decreased the activity of metalloproteinases and the serine protease family, the plasminogen urokinase activator (uPA) system. uPA interacts with specific receptors (uPAR) via domains such as epidermal growth factor (EGF) in the aminoterminal urokinase (ATF) fragment. This effect of curcumin causes inhibition of endothelial cell migration and stimulation of bFGF, transforming growth factor (TGF), TNF, hepatocyte growth factor (HGF), and VEGF release. Curcumin can also inhibit the expression of MMP-2 which acts through the FGF-2 angiogenic signaling pathway. The inhibitory effect of curcumin was also described for mouse keratinocytes. Curcumin reduces TGF-1-induced uPA levels in transformed keratinocytes and TGF- $\beta$-induced fibronectin synthesis as well as inhibits TGF--induced cell migration and stimulation and pterygium invasion. (Song et al., 2011; Dorota et al., 2019) 
Further studies revealed that curcumin induces TGF- $\beta$-induced factor (TGIF), which is a negative regulator of TGF- $\beta$ signaling. The results of the TGIF study prove the important role of TGIF in suppressing TGF- $\beta$ which plays a role in proliferation. (Song et al., 2011).

\section{Reference}

Coroneo M.T., Florakis G.J., Goins K.M., Hammersmith K. and Reidy J.J. 2019. External Disease and Cornea Section 8, San Francisco: American Academy of Ophthalmology.

Hasemi H., Mehdi K., Abbasali Y., Jafarzadehpour E., Ostadimoghaddam H. and Kangarif H. 2016. The Prevalence and Determinants of Pterigium in Rural Areas. J Curr Ophthalmol. Sep; 29(3): 194-198.

Hovanesian J.A., Starr C.E., Vroman D.T., Mah F.S., Gomes J.A., Farid M., Shamie N., Davidson R.S., John T., Holland E.J. and Kim T. 2017. Surgical Techniques and Adjuvants for The Management of Primary and Recurrent Pterygia. J Cataract Refract Surg. Mar;43(3):405-419.

Nuzzi R and Tridico F. 2018. How to Minimize Pterygium Recurrence Rates: Clinical Perspectives. Clin Ophthalmol. Nov 19;12:2347-2362.

Kim K.W., Park S.H. and Kim J.C. 2016. Fibroblast Biology in Pterygia. Exp Eye Res. Jan;142:32-9.

Dushku N., John M.K., Schultz G.S. and Reid T.W. 2001. Pterygia Pathogenesis: Corneal Invasion by Matrix Metalloproteinase Expressing Altered Limbal Epithelial Basal Cells. Arch Ophthalmol. May;119(5):695-706.

Cárdenas-cantú E., Zavala J., Valenzuela J. and Valdez-garcía J.. 2016. Molecular Basis of Pterigium Development. Semin Ophthalmol.;31(6):567-83.

Horbelt D., Denkis A. and Knaus P.. 2012. A portrait of Transforming Growth Factor $\beta$ Superfamily Signalling: Background Matters. The Int J of Biochem \& Cell Bio 44(3):469-74

Zada M., Pattamatta U. and White A. 2017. Modulation of Fibroblasts in Conjunctival 
Wound Healing. Ophthalmology. Feb;125(2):179-192.

Ellis S., Lin E.J. and Tartar D. 2018. Immunology of Wound Healing. Curr Dermatol Rep . 2018;7(4):350-358.

Nakerakanti S. and Trojanowska M. 2012. The Role of TGF- $\beta$ Receptors in Fibrosis. Open Rheumatol J; 6:156-62.

Hu H. H., Chen D.Q., Wang Y.N., Feng Y.L., Cao G., Vaziri N.D. and Zao Y.Y. 2018. New Insights Into TGF- $\beta$ /Smad Signaling in Tissue Fibrosis. Chem Biol Interact. Aug 25;292:7683.

Chui J., Di-Girolamo N., Wakefield D. and Coroneo M.T. 2008. The Pathogenesis of Pterigium. Current Concepts and Their Therapeutic Implications. Ocul Surf. Jan;6(1):24-43.

Dorota M.R., Anna O.L., Anna H., Agata G., Anna M. D., Piotr S. 2019. Therapeutic Potential of Curcumin in Eye Diseases. Cent Eur J Immunol.; 44(2): 181-189.

Stati G., Sancilio S., Basile M., Angelini A., Pietro R.D. 2020. Curcuma Longa Aqueous Extract: a Potential Solution for The Prevention of Corneal Scarring as a Result of Pterygium Surgical Excision (Review). Int J Mol Med. Dec;46(6):1951-1957.

Song K., Peng S., Sun Z., Li H. and Yang R. 2011. Curcumin Suppresses TGF- $\beta$ Signaling by Inhibition of TGIF Degradation in Scleroderma Fibroblasts. Biochem Biophys Res Commun. Aug 12;411(4):821-5. 